\title{
The precursors and impacts of BSR on AMT acquisition and implementation.
}

\begin{abstract}
This paper reports on the results of research into the connections between transaction attributes and buyer-supplier relationships (BSRs) in advanced manufacturing technology (AMT) acquisition and implementation. The investigation began by examining the impact of the different patterns of BSR on the performance of the AMT acquisition. In understanding the phenomena, the study drew upon and integrated the literature of transaction cost economics theory, BSRs, and AMT, and used this as the basis for a theoretical framework and hypotheses development. This framework was then empirically tested using data that were gathered through a questionnaire survey with 147 companies and analyzed using a structural equation modeling technique. The results of the analysis indicated that the higher the level of technological specificity and uncertainty, the more firms are likely to engage in a stronger relationship with technology suppliers. However, the complexity of the technology being implemented was associated with BSR only indirectly through its association with the level of uncertainty (which has a direct impact upon BSR). The analysis also provided strong support for the premise that developing strong BSR could lead to an improved performance in acquiring and implementing AMT. The implications of the study are offered for both the academic and practitioner audience.
\end{abstract}

Keyword: Advanced manufacturing technology (AMT); Buyer-supplier relationships (BSRs); Malaysia; Technology implementation; Transaction cost economics (TCE) theory. 